\title{
Region-Dependent Differences and Alterations of Protective Thiol Compound Levels in Cultured Astrocytes and Brain Tissues
}

\author{
Tatsumi Adachi, ${ }^{*, a, b, c}$ Masahiko SAtoh, ${ }^{a, d}$ Rocky Pramanik, ${ }^{a}$ Sakiko Kuroda, ${ }^{a}$ Masami Ishido, ${ }^{a}$ and \\ Manabu Kunimoto ${ }^{a, e}$ \\ ${ }^{a}$ National Institute for Environmental Studies; 16-2 Onogawa, Tsukuba, Ibaraki 305-8506, Japan: ${ }^{b}$ Department of Basic \\ Medical Sciences, National Institute for Minamata Disease; 4058-18 Hama, Minamata, Kumamoto 867-0008, Japan: \\ ${ }^{c}$ Faculty of Pharmaceutical Sciences, Chiba Institute of Science; 15-8 Shiomi-cho, Choshi, Chiba 288-0025, Japan: \\ ${ }^{d}$ Gifu Pharmaceutical University; Mitahora-higashi, Gifu 502-8585, Japan: and ${ }^{e}$ Department of Public Health, School of \\ Pharmaceutical Sciences, Kitasato University; 5-9-1 Shirokane, Minato-ku, Tokyo 108-8641, Japan. \\ Received January 20, 2006; accepted April 8, 2006; published online April 11, 2006
}

\begin{abstract}
We examined region-dependent differences and alterations in the levels of protective thiol compounds, glutathione (GSH) and metallothionein (MT)-I and -II, in cultured rat astrocytes under several culture conditions and in brain tissues of rats at postnatal and weaning periods. Regardless of culture conditions, both protein concentrations and mRNA expressions of MT-I and -II were much higher in the cerebral hemisphere than in cerebellar astrocytes, whereas no difference was observed in GSH concentration. In both astrocytes, the GSH concentrations did not change within $12 \mathrm{~h}$ but significantly increased $24 \mathrm{~h}$ after being maintained in a serum-free defined medium. At $24 \mathrm{~h}$, protein concentrations and mRNA expressions of MT-I and -II also increased in the respective astrocytes, and were further enhanced when maintained in the presence of $50 \mu_{\mathrm{M}} \mathrm{Zn}^{2+}$. In the brain tissues, the MT-I/-II protein concentrations were significantly higher in the cerebral cortex (a part of the cerebral hemisphere) than in the cerebellum, whereas the GSH concentration was similar at both postnatal day (P) 1 and $P 35$. In addition, the concentrations in the respective regions were significantly higher at $P 35$ than at P1. These results suggest that region-dependent differences in the cellular levels of GSH and MTs in cultured astrocytes might reflect the in vivo differences, and that the levels of the respective thiol compounds in cultured astrocytes increase after serum elimination along with the region-dependent differences.
\end{abstract}

Key words glutathione; metallothionein; astrocyte; brain; regional difference

Astrocytes play many important roles such as maintaining neuronal survival and functions. ${ }^{1,2)}$ Therefore, it has been suggested that an astrocyte dysfunction may indirectly reinforce the neurotoxicity induced by some chemicals including mercury compounds, methylmercury (MeHg) and $\mathrm{Hg}^{2+}$, through disturbing ion homeostasis and inhibiting glutamate uptake into the astrocytes. ${ }^{3-5)}$ Since $\mathrm{MeHg}$ neurotoxicity is observed at relatively local regions, $\left.{ }^{6}\right)$ we hypothesized that this might reflect brain region-specific susceptibility to mercury compounds in astrocytes. However, regardless of culture conditions, no differences between astrocytes from the cerebral hemisphere and cerebellum were observed not only in susceptibility but also in mercury accumulation for the first few hours after exposure to $\mathrm{MeHg}$ or $\mathrm{Hg}^{2+}{ }^{2}$ ) In addition, we found that both susceptibility and mercury accumulation decreased with elapsed time after serum elimination until exposure. ${ }^{7)}$ Although the differences in susceptibility to these compounds could, therefore, be explained by those in mercury accumulation, it might be possible that some factors related with cellular defense against their toxicity build up with time after serum elimination. It has been demonstrated that some thiol compounds such as glutathione (GSH), an ubiquitous thiol-containing tripeptide, and metallothioneins (MTs), cysteine-rich low molecular weight proteins, are pivotal factors in protecting against the toxicity of mercury compounds in cultured astrocytes. For example, GSH can protect against the acute cytotoxicity of both $\mathrm{MeHg}$ and $\left.\mathrm{Hg}^{2+},{ }^{8}\right)$ and MTs induced by $\mathrm{Zn}^{2+}$ can also do so against that of MeHg. ${ }^{9}$ In addition, since MT-I and -II in cultured astrocytes are induced by metals including $\mathrm{Hg}^{2+}$ at higher doses than in cultured hepatocytes, ${ }^{10,11)}$ these MT isoforms would be able to protect against the toxicity of $\mathrm{Hg}^{2+}$ as observed in cultured hepatocytes. ${ }^{12)}$

In the present study, we examined alterations after serum elimination and region-dependent differences in the cellular levels of the protective thiol compounds, GSH and MTs, in cultured astrocytes prepared from the cerebral hemisphere and cerebellum. In addition, to clarify the significance of these alterations in cultured astrocytes, we also investigated the changes and brain region-dependent differences in these levels in vivo.

\section{MATERIALS AND METHODS}

Animals Male and female Wistar rats obtained from

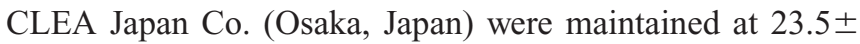
$1.5^{\circ} \mathrm{C}$ and $55 \pm 10 \%$ relative humidity under a 12 -h light cycle. The animals were given standard laboratory chow and tap water ad libitum. Pregnant rats were prepared as previously described, ${ }^{7,13)}$ and housed individually until birth. The animals received humane care throughout the experiment according to the Guidelines of the National Institute for Environmental Studies (NIES) and those of the National Institute for Minamata Disease (NIMD).

Cell Cultures Astrocyte cultures were prepared from the cerebral hemisphere and cerebellum of newborn rats (within $24 \mathrm{~h}$ after birth) as previously described. ${ }^{7)}$ In brief, the two brain regions, with the meninges carefully dissected off, were separately washed in $\mathrm{Ca}^{2+}, \mathrm{Mg}^{2+}$-free Hanks' balanced salt solution and treated with $0.1 \%$ trypsin at $37^{\circ} \mathrm{C}$ for $10 \mathrm{~min}$. Cells obtained were suspended in Basal Medium Eagle's with Earl's salts supplemented with $15 \%$ fetal calf serum (FCS; 
Invitrogen Co., Carlsbad, CA, U.S.A.), 0.1\% L-glutamine, $0.6 \%$ D-glucose, antibiotics (Penicillin-Streptomycin; Invitrogen Co.), and an antimycotic (Fungizon; Invitrogen Co.). The culture flasks $\left(25 \mathrm{~cm}^{2}\right.$; BD Bioscience; Billerica, MA, U.S.A.), into which cells were plated, were incubated at $37^{\circ} \mathrm{C}$ in $6 \% \mathrm{CO}_{2}$ in a humidified atmosphere until cells reached confluence. After removing floating cells that came off by shaking, the remaining cells, most of which were glial fibrillary acidic protein-positive astrocytes, ${ }^{7)}$ were collected using $0.25 \%$ trypsin, and resuspended with the $15 \%$ FCScontaining fresh medium. They were then plated on 6-well plates (BD Bioscience), and used for determining thiol compound levels after the plates reached $85-95 \%$ confluence.

Assays for GSH and MT-I/-II Proteins In some astrocyte cultures, the $15 \%$ FCS-containing medium was changed to a serum-free defined medium (SFDM), consisting of minimum essential medium with bovine serum albumin (BSA; $1 \mathrm{mg} / \mathrm{ml}$; Sigma, St. Louis, MO, U.S.A.), bovine apo-transferrin $(100 \mu \mathrm{g} / \mathrm{ml}$; Sigma $)$, insulin $(10 \mu \mathrm{g} / \mathrm{ml}$; Sigma $)$, aprotinin ( $1 \mu \mathrm{g} / \mathrm{ml}$; Sigma), thyroxine (0.1 nm; Sigma), and antibiotics (Penicillin-Streptomycin; Invitrogen Co.), as previously described. ${ }^{7)}$ Three, 12 , and $24 \mathrm{~h}$ after the medium change, or without this change, cells were rinsed 3 times with $\mathrm{Ca}^{2+}$, $\mathrm{Mg}^{2+}$-free phosphate buffered saline [PBS $\left.(-)\right]$ and then deproteinized using 5\% perchloric acid containing $1 \mathrm{~mm}$ ethylenediamine- $N, N, N^{\prime}, N^{\prime}$-tetraacetate (EDTA). They were immediately centrifuged and divided into supernatants and pelleted materials. Total GSH in each supernatant was determined by the method of Tietze. ${ }^{14)}$ After the pellets were dissolved in $1 \mathrm{~N} \mathrm{NaOH}$, whole protein content was determined by the method of Lowry et al. ${ }^{15)}$ using BSA as a standard.

Other cells used for MT assay were divided into 3 groups. Before collecting the cells, two groups were maintained in

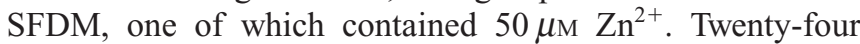
hours after being maintained in SFDM with or without $\mathrm{Zn}^{2+}$, or without the medium change, cells were rinsed 3 times with PBS (-), and then collected. The content of MT-I/-II proteins in each sample was determined by radioimmunoassay ${ }^{16}$ with modifications. ${ }^{17)}$ Whole protein content was determined as described above.

Male Wistar rats aged 1 and $35 \mathrm{~d}$ were used to determine the levels of the thiol compounds in brain tissues. Each rat was anesthetized using ether, and the brain was excised. Each brain on postnatal day (P)35 was then cut in half, and from one part the cerebral cortex (neocortex) and cerebellum were obtained. P1 rats were separated into two groups, from one of which the whole brain and cerebral cortex were obtained, while from the other we obtained the cerebral cortex and cerebellum. For GSH assay, the tissues were immediately deproteinized using 5\% perchloric acid containing $1 \mathrm{~mm}$ EDTA, and then centrifuged to obtain supernatants. For MT assay, the tissues from another group of rats were homogenized in $10 \mathrm{~mm}$ tris(hydroxymethyl)aminomethane (Tris)- $\mathrm{HCl}$ buffer ( $\mathrm{pH}$ 7.4). The contents of total GSH in the supernatants and MT-I/-II proteins in the homogenates were determined as described above.

Northern Blotting The probes for Northern blotting of rat MT-I and -II were prepared using reverse transcriptionpolymerase chain reaction (RT-PCR). A male Wistar rat (approximately $350 \mathrm{~g}$ ) was intraperitoneally administered zinc sulfate $(18.7 \mathrm{mg} \mathrm{Zn} / \mathrm{kg})$ to induce MT-I and -II mRNA.
Twenty-four hours after the administration, the rat was perfused with ice-cold saline from the heart under ether anesthesia, and the liver was then excised. Total RNA was prepared from the rat liver using an RNeasy kit (Quigen GmbH., Hilden, Germany) according to the manufacturer's instructions. Poly $\mathrm{A}^{+}$was concentrated with biotin-labeled magnetic beads. Poly $\mathrm{A}^{+}$RNA was reverse transcribed with oligo dT primer (Invitrogen Co.) and reverse transcriptase (Invitrogen Co.). The reverse transcribed product was used as a PCR template with the following various sets of PCR primers: MT-I: sense, CTTCTTGTCGCTTACACCGTTGCTC, anti-sense, CATGCTCGGTAGAAAACGGGGTTTAG, ${ }^{18)}$ and MT-II: sense, CTCCTAGAACTCTACAGCGATCTCTC, anti-sense, GAAAAAAGTGTGGAGAACCGGTCAGG. ${ }^{19}$ ) The PCR products were gel-purified and confirmed by direct sequencing.

Total RNA in cultured astrocytes was prepared as described above $24 \mathrm{~h}$ after being maintained in SFDM with or without $50 \mu \mathrm{m} \mathrm{Zn}^{2+}$, or without the medium change. RNA was separated by electrophoresis in a $1 \%$ gel containing $6.7 \%$ formaldehyde, and was transferred to a nylon membrane (Hybond-N+; Amersham Biosciences Co., Piscataway, NJ, U.S.A.) with $10 \times \operatorname{SSPE}\left(0.15 \mathrm{M} \mathrm{NaCl}, 0.01 \mathrm{M} \mathrm{NaH} \mathrm{PO}_{4}\right.$, and $1 \mathrm{~mm}$ EDTA, $\mathrm{pH} 7.4$ ) as the transferring solution as previously described. ${ }^{20}$ ) The membrane was pre-hybridized at $68^{\circ} \mathrm{C}$ for $2-3 \mathrm{~h}$ in Express hybridization solution (BD Bioscience Clontech, Palo Alto, CA, U.S.A.) containing $200 \mathrm{mg} / \mathrm{ml}$ denatured salmon sperm DNA. cDNA fragments of rat MT-I and -II were labeled with $\left[\alpha_{-}{ }^{32} \mathrm{P}\right] \mathrm{dCTP}$ (Amersham Biosciences Co.) and the random primer DNA labeling kit version 2 (Takara Bio Co., Shiga, Japan). The radioactive probe was added to the pre-hybridization solution and incubated for $12-16 \mathrm{~h}$ at $68^{\circ} \mathrm{C}$. The membrane was washed with $2 \times \mathrm{SSPE} / 0.1 \%$ sodium dodecylsulfate $(\mathrm{SDS})$ and $0.1 \times \mathrm{SSPE} /$ $0.1 \%$ SDS at $50{ }^{\circ} \mathrm{C}$. The hybridized cDNA was located, and radioactive cDNA was quantified by exposure to a Bio-Imaging plate and analyzed with a FUJIX Bio-Imaging Analyzer BAS 2000 (Fuji Photo Film, Tokyo, Japan).

Statistical Analysis Significant differences between individual means were determined by one-way analysis of variance (ANOVA) followed by Duncan's new multiple range test or by Student's $t$-test. Differences were considered significant at $p<0.05$.

\section{RESULTS}

Figures 1 and 2 show cellular levels of protective thiol compounds, GSH and MTs, respectively, in cultured astrocytes from the cerebral hemisphere and cerebellum under several culture conditions. In the presence of serum, no difference was observed in total GSH concentrations between astrocytes from both regions (Fig. 1). In both astrocytes, the concentrations did not change within $12 \mathrm{~h}$, but significantly increased $24 \mathrm{~h}$ after serum elimination (Fig. 1). Even at that time, however, no region-dependent difference was observed (Fig. 1). In contrast to the GSH levels, MT-I/-II protein concentrations were significantly higher in the cerebral hemisphere than in cerebellar astrocytes under all conditions examined (Fig. 2A). These protein concentrations more than doubled $24 \mathrm{~h}$ after serum elimination (Fig. 2A, nos. 3 and 4), compared with those in the respective astrocytes maintained in the 15\% FCS-containing medium (Fig. 2A, nos. 1 and 2). 
In the presence of serum, expression levels of MT-I and -II mRNA, which normalized the expression of glyceraldehyde3-phosphate dehydrogenase (GAPDH) mRNA, in cerebellar astrocytes were $17 \%$ and $13 \%$ of those in cerebral hemisphere astrocytes, respectively (Fig. 2B, lanes 1 and 4). Both MT-I and -II mRNA expressions increased approximately two-fold $24 \mathrm{~h}$ after serum elimination in cerebral hemisphere astrocytes (Fig. 2B, lanes 1 and 2), while increasing approximately 4 and 10 times in cerebellar astrocytes (Fig. 2B, lanes 4 and 5), respectively. In the astrocytes from the respective regions, protein concentrations (Fig. 2A, nos. 3-6) and mRNA expressions (Fig. 2B, lanes 2, 3, 5 and 6) of MT-I and -II were much higher with exposure to $50 \mu \mathrm{m} \mathrm{Zn}^{2+}$ than in those without it, suggesting that region-dependent differences in the MT levels might be maintained regardless of culture conditions, even after induction by $\mathrm{Zn}^{2+}$.

To clarify the significance in these alterations observed in cultured astrocytes, total GSH and MT-I/-II proteins were examined in the whole brain, cerebral cortex (a part of the cerebral hemisphere) and cerebellum of P1 and P35 rats. The concentrations of total GSH (Fig. 3A) and MT-I/-II proteins (Fig. 3B) in all of the respective brain regions examined were

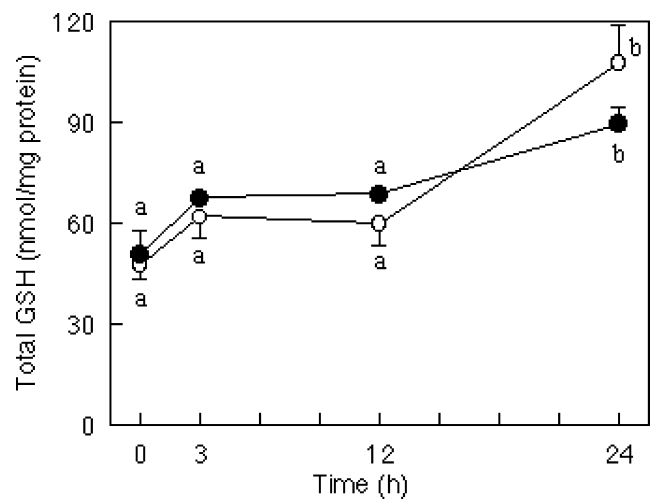

Fig. 1. Time-Dependent Changes in Total GSH Concentrations in Cultured Astrocytes

Astrocytes prepared from the cerebral hemisphere $(\bigcirc)$ or cerebellum $(\bigcirc)$ were maintained in SFDM for $0-24 \mathrm{~h}$. Values represent the mean \pm S.D. obtained from 3 determinations. Values with different letters (a and $b)$ indicate significant differences $(p<0.05)$. significantly higher at P35 (nos. 5-7) than at P1 (nos. 14). At both times, the total GSH concentration in the cerebral cortex (nos. 2, 3 and 6) was similar to that in the cerebellum (nos. 4,7 ) as well as that in the whole brain (nos. 1 and 5) (Fig. 3A). In contrast, the MT-I/-II protein concentrations in the cerebral cortex (nos. 2, 3 and 6) were significantly higher than in the cerebellum (nos. 4 and 7) in both P1 and P35 rats, although they were similar to those in the whole brain (nos. 1 and 5) (Fig. 3B).
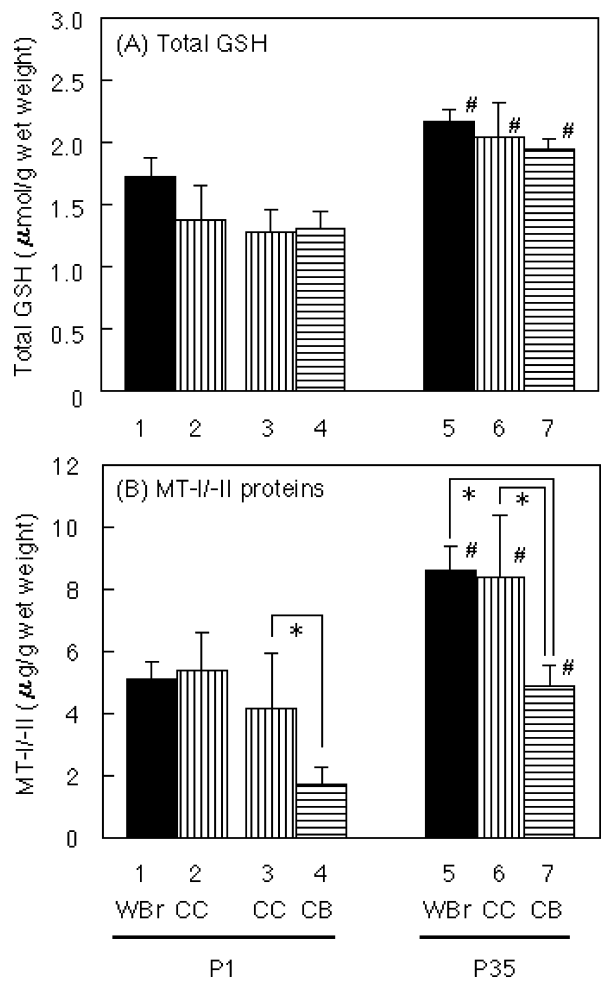

Fig. 3. Concentrations of Total GSH and MT-I/-II Proteins in Brain Tissues of P1 and P35 Rats

Abbreviations of brain regions: $\mathrm{WBr}$, whole brain; $\mathrm{CC}$, cerebral cortex; $\mathrm{CB}$, cerebellum. Values represent the mean \pm S.D. obtained from 4 to 5 rats. $(*)$ indicates significant differences between different brain regions at the specified ages $(p<0.01)$. (\#) indicates significant differences from $\mathrm{P} 1$ rats in the specified brain regions $(p<0.01)$.
(A) MT-Ii-II proteins

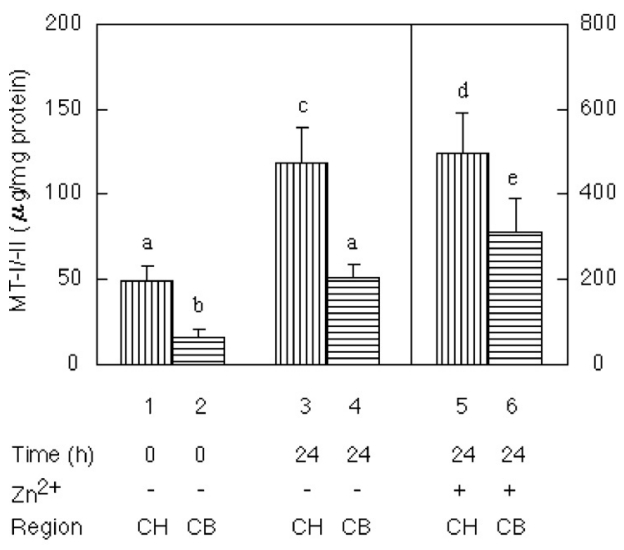

(B) $M T-1$ and $-I I$ mFNA.

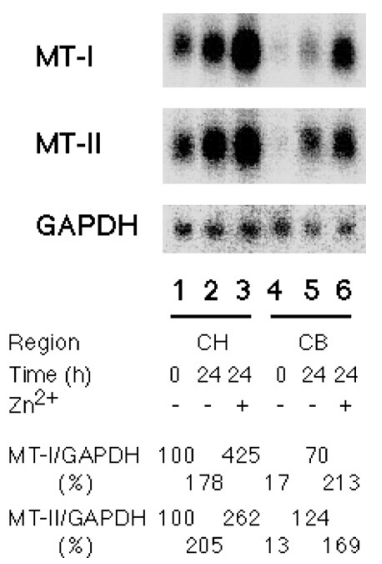

Fig. 2. Protein Concentrations and mRNA Expressions of MT-I and -II in Cultured Astrocytes

Astrocytes prepared from the cerebral hemisphere (CH) or cerebellum (CB) were maintained in SFDM with or without of $50 \mu \mathrm{M} Z \mathrm{n}^{2+}$ for 0 or $24 \mathrm{~h}$. (A) MT-I/-II protein concentrations. Values represent the mean \pm S.D. obtained from 3 determinations. Values with different letters $(\mathrm{a}-\mathrm{e})$ indicate significant differences $(p<0.05)$. (B) mRNA expressions of MT-I and -II. MT-I and -II mRNA expressions were normalized to the expression of GAPDH mRNA, and expressed as percentages in relation to the ratio (MT-I/GAPDH or MTII/GAPDH) in the cerebral hemisphere astrocytes in the presence of serum (lane 1). 


\section{DISCUSSION}

In the present study, there were region-dependent differences in concentrations of MT-I/-II proteins both in vivo and in cultured astrocytes. The MT-I/-II protein concentrations in vivo were higher in the cerebral cortex (a part of the cerebral hemisphere) than in the cerebellum at both P1 and P35 (Fig. 3B). In addition, the concentrations of these MT isoforms were higher in the cerebral hemisphere (including the cerebral cortex) than in cerebellar astrocytes (Fig. 2A). In contrast, no region-dependent difference in the GSH concentration was observed either in vivo (Fig. 3A) or in cultured astrocytes (Fig. 1). It has been demonstrated that MT-I and -II are mainly expressed in glial cells such as astrocytes rather than in neurons. ${ }^{10,11)}$ In addition, GSH levels appear to be higher in astrocytes than in neurons both in vivo and in culture. ${ }^{21)}$ Moreover, since GSH released by astrocytes is provided to neurons and used as a source of neuronal $\mathrm{GSH}^{21,22)}$ an alteration in the GSH metabolism in astrocytes would be reflected by the neuronal GSH concentration. Accordingly, region-dependent differences in these concentrations in cultured astrocytes may in part reflect those in the in vivo levels of astrocytes. We also found in this study that the concentrations of GSH and MT-I/-II proteins increased in the respective astrocytes after serum elimination (Figs. 1, 2A), and those in the respective brain regions in vivo were higher at the weaning than at the postnatal period (Figs. 3A, B). Although the reasons for the increases in cultured astrocytes after serum elimination remain uncertain, it is possible that cultured astrocytes maintained in SFDM would undergo maturation, as demonstrated in some previous reports, ${ }^{23,24)}$ in spite of some differences in culture conditions. Therefore, one possibility is that the increases in cultured astrocytes might result from their maturation. In addition, serum-deprivation stress might partly contribute to the increases. To clarify why these levels increase after serum elimination, further study in detail would be necessary.

We recently found that susceptibility was higher in cultured astrocytes exposed to $\mathrm{MeHg}$ or $\mathrm{Hg}^{2+}$ immediately after serum elimination rather than in those exposed $24 \mathrm{~h}$ after. ${ }^{7}$ Since concentrations of GSH and MT-I/-II proteins, which could preserve the toxicity of mercury compounds in cultured astrocytes, ${ }^{8,9)}$ increased in the respective astrocytes at least $24 \mathrm{~h}$ after serum elimination (Figs. 1, 2A), such alterations might in part explain the lower susceptibility to mercury compounds in astrocytes maintained in SFDM for $24 \mathrm{~h}$. We recently reported, on the other hand, that accumulation of $\mathrm{Hg}^{2+}$ for $24 \mathrm{~h}$ was higher in the cerebral hemisphere than in cerebellar astrocytes when it was exposed both immediately and $24 \mathrm{~h}$ after serum elimination, although that for $3 \mathrm{~h}$ was similar between astrocytes from both regions. ${ }^{7)}$ In contrast, no region-dependent difference in $\mathrm{MeHg}$ accumulation for 3 or $24 \mathrm{~h}$ was observed, except for that for $24 \mathrm{~h}$ exposed $24 \mathrm{~h}$ after serum elimination. ${ }^{7)}$ In the present study, the MT-I/-II protein concentrations were higher in the cerebral hemisphere than in cerebellar astrocytes regardless of culture conditions, even after being maintained in the presence of $\mathrm{Zn}^{2+}$ (Fig. 2A), although the region-dependent differences have not been determined after exposure to mercury compounds. It is well known that MTs bind to $\mathrm{Hg}^{2+}$ but hardly to MeHg. ${ }^{25-27)}$ In addition, it is possible that $\mathrm{Hg}^{2+}$ binds mainly to high molecular weight proteins at $3 \mathrm{~h}$, and the binding shifts to MTs at $24 \mathrm{~h}$. Accordingly, the region-dependent differences in accumulation of $\mathrm{Hg}^{2+}$ but not $\mathrm{MeHg}$ for $24 \mathrm{~h}$ might reflect those in MT-I/-II protein concentrations. Thus, the alterations and differences in the cellular levels of these thiol compounds might in part explain those in the susceptibility to the toxic metals such as mercury compounds and in their retention in cultured astrocytes.

The present results suggest that region-dependent differences in the cellular levels of GSH and MTs in cultured astrocytes might reflect the in vivo differences, and be maintained regardless of culture conditions. It is also suggested that, in cultured astrocytes, the levels of these thiol compounds increase after serum elimination, and these alterations might modulate the metal toxicity.

Acknowledgments The authors wish to thank Dr. Chiharu Tohyama (NIES) and Dr. Atsuhiro Nakano (NIMD) for their encouragement. We are also grateful to Ms. Ryuko Takenoshita-Shimoda (NIMD), Ms. Kyoko Yamada (NIMD) and Ms. Rieko Ochiai (NIMD) for their technical assistance.

\section{REFERENCES}

1) Manthorpe M., Pettmann B., Varon S., "The Biochemical Pathology of Astrocytes," ed. by Norenberg M. D., Hertz L., Schousboe A., Alan R. Liss, New York, 1988, pp. 41-58.

2) Vernadakis A., Prog. Neurobiol., 49, 185-214 (1996).

3) Brookes N., J. Neurochem., 50, 1117-1122 (1988).

4) Brookes N., Kristt D. A., J. Neurochem., 53, 1228-1237 (1989).

5) Aschner M., Eberle N. B., Miller K., Kimelberg H. K., Brain Res., 530, 245-250 (1990).

6) World Health Organization (WHO), "Environmental Health Criteria 101, Methylmercury," WHO, Geneva, 1990.

7) Adachi T., Kunimoto M., Biol. Pharm. Bull., 28, 2308-2311 (2005).

8) Aschner M., Mullaney K. J., Wagoner D., Lash L. H., Kimelberg H. K., Brain Res., 664, 133-140 (1994).

9) Aschner M., Conklin D. R., Yao C. P., Allen J. W., Tan K. H., Brain Res., 813, 254-261 (1998).

10) Kramer K. K., Liu J., Choudhuri S., Klaassen C. D., Toxicol. Appl. Pharmacol., 136, 94-100 (1996).

11) Aschner M., Cherian M. G., Klaassen C. D., Palmiter R. D., Erickson J. C., Bush A. I., Toxicol. Appl. Pharmacol., 142, 229-242 (1997).

12) Liu J., Kershaw W. C., Klaassen C. D., Toxicol. Appl. Pharmacol., 107, 27-34 (1991)

13) Adachi T., Takanaga H., Kunimoto M., Asou H., J. Neurosci. Res., 79, $608-615$ (2005)

14) Tietze F., Anal. Biochem., 27, 502-522 (1969).

15) Lowry O. H., Rosebrough N. J., Farr A. L., Randall R. J., J. Biol. Chem., 193, 265-275 (1951).

16) Tohyama C., Shaikh Z. A., Fund. Appl. Toxicol., 1, 1-7 (1991).

17) Nishimura H., Nishimura N., Tohyama C., J. Histochem. Cytochem., 37, 715-722 (1989)

18) Anderson R. D., Birren B. W., Ganz T., Piletz J. E., Herschman H. R., $D N A, 2,15-22$ (1983)

19) Anderson R. D., Birren B. W., Piletz J. E., Herschman H. R., Mol. Cell. Biol., 6, 302-314 (1986).

20) Pramanik R., Ueno A., Nishikawa H., Nagata T., Inoue H., FEBS Lett., 393, 193-196 (1996); corrigendum FEBS Lett., 415, 352 (1997).

21) Dringen R., Gutterer J. M., Hirrlinger J., Eur. J. Biochem., 267, 4912 4916 (2000).

22) Dringen R., Pfeiffer B., Hamprecht B., J. Neurosci., 19, 562-569 (1999).

23) Weibel M., Pettmann B., Labourdette G., Miehe M., Bock E., Sensenbrenner M., Int. J. Dev. Neurosci., 3, 617-630 (1985).

24) Nelson R. B., Siman R., Dev. Brain Res., 54, 93-104 (1990).

25) Chen R. W., Ganther H. E., Hoekstra W. G., Biochem. Biophys. Res Commun., 51, 383-390 (1973).

26) Sato M., Sugano H., Takizawa Y., Arch. Toxicol., 47, 125-133 (1981).

27) Stillman M. J., Coord. Chem. Rev., 144, 461-511 (1995). 